\title{
Activation method for cross section measurements related to the p-process nucleosynthesis
}

\author{
Nalan Özkan* \\ Kocaeli University \\ Faculty of Science and Literature, Department of Physics, ,Umuttepe, Kocaeli TR-41380 \\ TURKEY \\ E-mail: nozkan@kou.edu.tr
}

\begin{abstract}
The 35 stable nuclei with the atomic number $Z \geq 34$ located at neutron deficient side of valley of beta-stability are called as $p$-nuclei and are produced by so called $p$-process. $P$-process nucleosynthesis proceeds mainly via a series of photon induced reactions $(\gamma, n),(\gamma, p)$, and/or $(\gamma, \alpha)$ in the astrophysical environment. A large reaction network involving thousands of reaction rates is required to describe the $p$-process nucleosynthesis. Since, so far most of these reactions are not accessible experimentally, these reaction rates mainly rely on the Hauser Feshbach statistical model. Most $\gamma$-induced reactions are very difficult to measure directly. As alternative methods, the charged particle induced cross sections can be measured by activation method and on-line $\gamma$-detection method, and calculated from the inverse reaction cross sections using the detailed balance theorem. Therefore, cross section measurements for proton and alpha induced reactions are crucial for the $p$-process nucleosynthesis and for testing the recent statistical model predictions of Hauser-Feshbach. The primary aim of this paper is to describe the status of experimental cross section measurements of radiative charged particle capture reactions. The experimental database obtained by activation method up to now and what can be done in the future for the $p$-process are given.
\end{abstract}

International Symposium on Nuclear Astrophysics - Nuclei in the Cosmos - IX

25-30 June, 2006

CERN, Geneva, Switzerland

\footnotetext{
${ }^{*}$ Speaker.
} 


\section{Introduction}

The heavy nuclei with $\mathrm{Z} \geq 26$ are mainly produced by distinct nucleosynthetic mechanisms known as s-, $r$-, and $p$-processes. The $p$-process is responsible for the production of the observed rare abundances of 35 proton rich stable isotopes between $\mathrm{Se}$ and $\mathrm{Hg}$ (see Fig. 1). These socalled $p$-nuclei are shielded by stable nuclei from the production via the $s$ - and $r$-process. It is believed that the $p$-process, mainly, proceeds via a combination of photodisintegration reactions, $(\gamma, n),(\gamma, p)$ and $(\gamma, \alpha)$ on an existing heavy s- and $r$-seed in the temperature range of 2$3 \times 10^{9} \mathrm{~K}$. Initially, the nuclides are driven by a sequence of $(\gamma, n)$ reactions to the proton rich side since the binding energy of neutrons becomes gradually larger along the isotopic path. The reaction path is then deflected by the $(\gamma, p)$ and/or $(\gamma, \alpha)$ reactions, and the reaction flow path will branch. Finally, it is complemented by some $\beta^{+}$, electron captures, and $(n, \gamma)$ reactions [1]. The $p$-process and possible $p$-process sites have been reviewed in detail by Arnould and Goriely [2].

In comparison to the $s$ - and $r$-process, not much study is devoted to the $p$-process although a large reaction network of several thousands of reaction rates involving stable as well as unstable proton rich nuclei within a given astrophysical environment is required to describe the $p$-process nucleosynthesis. The determination of cross sections at relevant temperatures via direct or inverse measurements is difficult due to the fact that the corresponding energies are well below the Coulomb barrier so that the cross sections are extremely small. Another difficulty is that the $p$-nuclei have very little natural abundances $(0.01 \%-1 \%)$ and that measurements require highly enriched targets. Hence, not much associated reaction rates have been measured above Fe. The $p$-process studies are based mostly on Hauser-Feshbach statistical models to predict the reaction rates. Comparison with the statistical models has shown that the global statistical model approaches work well at stability for neutron and proton capture reactions within less than a factor of two, but that there are problems predicting alpha capture reactions due to the uncertainty in global optical alpha potentials $[3,4]$. It is, therefore, crucial to investigate the reaction cross sections experimentally in order to test the reliability of the input parameters (e.g., optical potentials). Recently, there has been increased interest in measuring the cross sections of charged particle induced reactions, as explained in the next section.

\section{Experimental Situation for Charged Particle Induced Reactions}

Most $\gamma$-induced reactions are very difficult to measure directly. There are a few measurements of photodisintegration reaction cross sections related to $p$-process by using Bremsstrahlung photons produced by stopping electrons in a radiator material or using laser inverse-Compton scattering photons produced by head-on collisions of laser photons on relativistic electrons. Even though the investigation of $(\gamma, n)$ reactions for selected nuclei $(Z>73)$ along the $p$-process path has been started, the investigation of $(\gamma, p)$ and $(\gamma, \alpha)$ reactions is still a challenging future project $[5,6]$.

More traditionally, the particle induced reaction cross sections are measured by two methods: activation method and on-line $\gamma$-detection method. Their inverse photodisintegration reaction cross sections are calculated using the detailed balance theorem. 


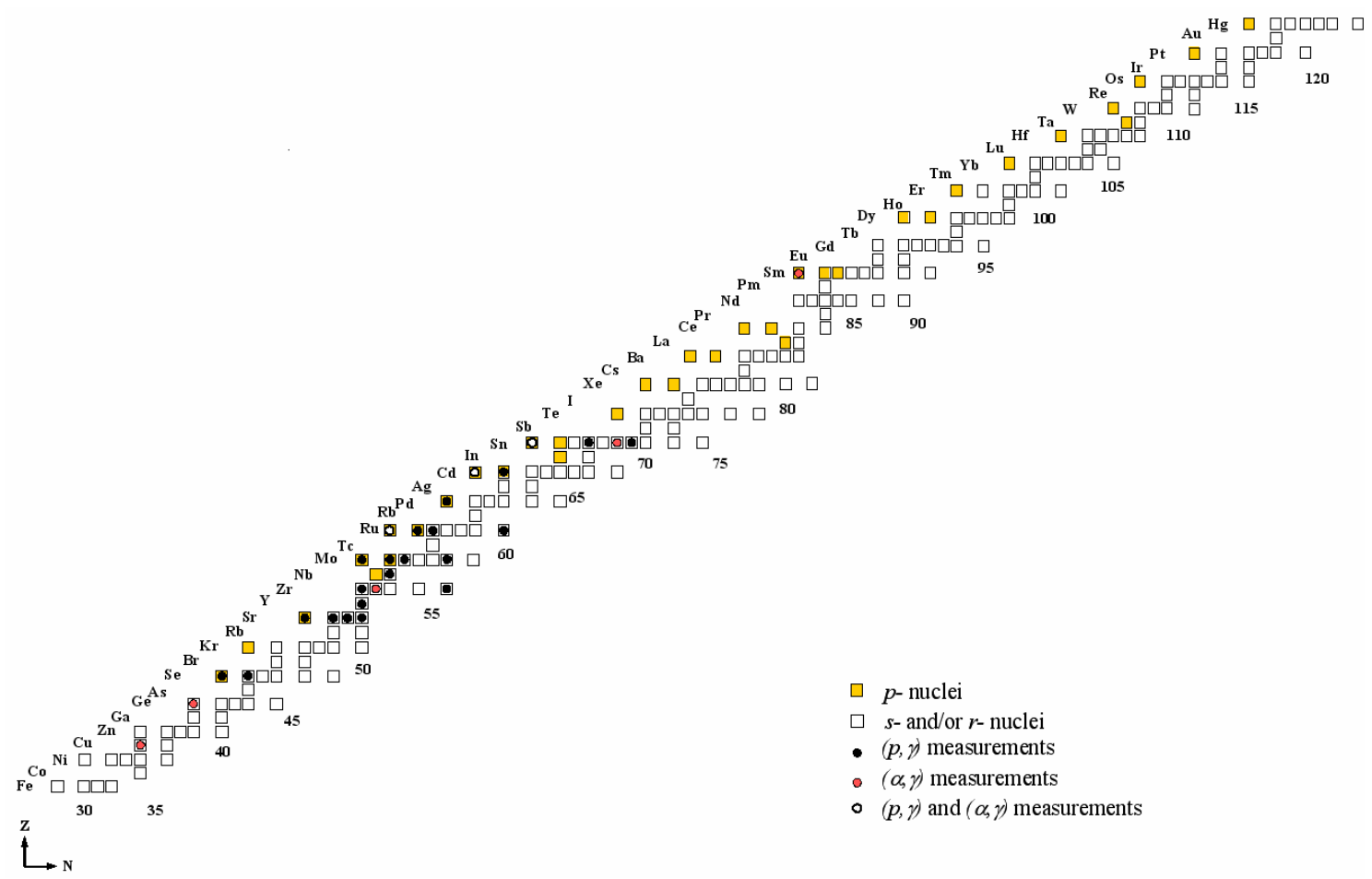

Figure 1: Part of the table of isotopes relevant to $p$-process. $35 p$-nuclei are shown with yellow boxes. Additionally, $(p, \gamma)$ and $(\alpha, \gamma)$ measurements are shown with black and red circles, respectively. Both $(p, \gamma)$ and $(\alpha, \gamma)$ measurements are represented by white circles.

If the particle induced reaction product is stable, the on-line $\gamma$-detection technique is the appropriate one. By using mostly an array of HPGe detectors detecting the individual transitions with $\gamma$-angular distributions or $4 \pi \mathrm{NaI}$ summing detectors detecting the sum of the energies of the cascading transitions, $(p, \gamma)$ reaction measurements on isotopes of ${ }^{88} \mathrm{Sr}[7],{ }^{89} \mathrm{Y}[8],{ }^{90} \mathrm{Zr}[9]$, ${ }^{93} \mathrm{Nb}[10]$ and $(\alpha, \gamma)$ reaction measurements on those of ${ }^{70} \mathrm{Ge}[11],{ }^{91} \mathrm{Zr}[12],{ }^{118} \mathrm{Sn}$ [12] have been studied.

On the other hand, the activation technique is an appropriate technique if the reaction product is radioactive. The half-lives should be comparable to the timescales of irradiation and counting times. First, the targets are bombarded with either a proton or alpha beam. Then, the number of unstable nuclei created, and thus the cross section, could be determined by measuring the radioactivity of the residual nucleus. The details for the activation method can be found in Ref. [13]. In the last decade, many proton capture reaction cross sections were studied by activation method, whereas limited numbers of $\alpha$-capture reaction cross sections, mostly for the low mass region, are available. The $(p, \gamma)$ reaction cross sections have been measured with the targets of ${ }^{74,76} \mathrm{Se}[14],{ }^{84,86,87} \mathrm{Sr}[15],{ }^{92,94,95,98} \mathrm{Mo} \mathrm{[16],}{ }^{96} \mathrm{Zr} \mathrm{[17],}{ }^{96,98,99,}{ }^{104} \mathrm{Ru}$ [18], ${ }^{102} \mathrm{Pd}[13]$, ${ }^{106,108} \mathrm{Cd}[19],{ }^{112,119} \mathrm{Sn}$ [17], and ${ }^{116} \mathrm{Sn}$ [13]. Experimental data for alpha induced reactions related to the $p$-process are even less than those for proton capture reactions. Alpha induced reaction cross sections, in the region of interest, on the targets of ${ }^{96} \mathrm{Ru}[20],{ }^{106} \mathrm{Cd}[21],{ }^{112} \mathrm{Sn}[13$, 22], and ${ }^{144} \mathrm{Sm}[23]$ have been investigated. The advantage of the activation method is the fact that it can be used to measure simultaneously the cross sections for different isotopes of an element by using natural targets as in the case of $(p, \gamma)$ measurements on $\mathrm{Se}$ [14], $\mathrm{Sr}$ [15], 
Mo [16] and $\mathrm{Ru}$ [18]. Since the irradiation and counting setups are in different areas, the shielding of the counting setup can be better, and thus the background is much less than that in on-line $\gamma$-detection technique. The new setups and methods that are able to provide the measurements on the targets with shorter half-lives (seconds to minutes) of the reaction products can be valuable next projects.

A synopsis of measured $(p, \gamma)$ and $(\alpha, \gamma)$ reaction cross sections in last two decades via the activation method and on-line $\gamma$-detection method at astrophysical energies is shown in Figure 1. Data are available only for stable targets up to about the Sn region. The experimental results, especially $(\alpha, \gamma)$ reaction cross sections, are frequently inconsistent with their model predictions. The experimental measurements for ${ }^{106} \mathrm{Cd},{ }^{112} \mathrm{Sn}$ and ${ }^{144} \mathrm{Sm}$ are lower than the theoretically predicted results of NON-SMOKER statistical model code [24]. The experimental $S$-factor values for the ${ }^{112} \mathrm{Sn}(\alpha, \gamma){ }^{116} \mathrm{Te}$ reaction [22] deviate in the lower energy range from the predictions of NON-SMOKER code as shown in Figure 2, as an example.

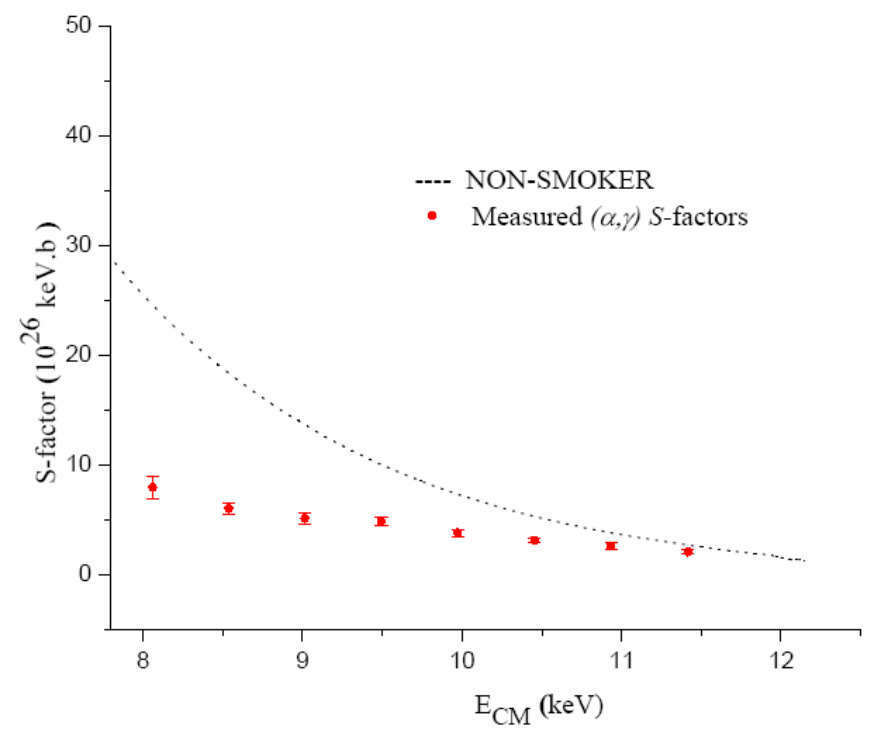

Figure 2: Theoretical NON-SMOKER code predictions [24] deviate in the lower energy range from the experimental $S$-factor values for the ${ }^{112} \operatorname{Sn}(\alpha, \gamma){ }^{116} \mathrm{Te}$ reaction as seen in Ref. [22].

More charged particle induced reaction cross section measurements especially on isotopes above $\mathrm{Sn}\left(\right.$ except $\left.{ }^{144} \mathrm{Sm}\right)$ as seen in Figure 1 are required for the statistical models of $(\gamma, p)$ and $(\gamma, \alpha)$ reaction rate calculations to be improved. Especially, $(\alpha, \gamma)$ measurements will affect the modeling of the alpha particle nucleus optical potentials. For this purpose, several measurements using activation method have been investigated in the collaboration with Kocaeli University (Turkey), the University of Notre Dame (USA) and ATOMKI (Hungary) [21, 22]. The results of $(\alpha, \gamma)$ measurements on ${ }^{106} \mathrm{Cd}$ and ${ }^{112} \mathrm{Sn}$ underline the necessity of the improvement on the optical $\alpha$ potentials. In order to determine a reliable global optical potential, recent efforts of elastic $\alpha$ scattering experiments on different isotopes are also in progress [25]. 
Acknowledgment is made to the Kocaeli University for the financial support, to the Scientific and Technical Research Council of Turkey (TUBITAK) - Grant TBAG-U/111 (104T2467), and to the University of Notre Dame and ATOMKI for the excellent collaboration.

\section{References}

[1] S. E. Woosley and W. M. Howard, Ap. J. Suppl. 36 (1978) 285.

[2] M. Arnould, S. Goriely, Phys. Rep. 384 (2003) 1.

[3] P. Descouvemont, T. Rauscher, Nucl. Phys. A 777 (2006) 137.

[4] D. Galaviz et al. Phys. Rev. C 71 (2005) 065802.

[5] K. Sonnabend et al. Phys. Rev. C 70 (2004) 035802.

[6] H. Utsunomiya et al. Nucl. Phys. A 777 (2006) 459.

[7] S. Galanopoulos et al. Phys. Rev. C 67 (2003) 015801.

[8] P. Tsagari et al. Phys. Rev. C 70 (2004) 015802.

[9] C. E. Laird et al. Phys. Rev. C Phys. 35 (1987) 1265.

[10] S. Harissopulos et al. Phys. Rev C 64 (2001) 055804.

[11] Zs. Fülop et al. Z. Phys. A 355 (1996) 203.

[12] S. Harissopulos et al. Nucl. Phys. A 758 (2005) 505.

[13] N. Özkan et al. Nucl. Phys. A 710 (2002) 469.

[14] Gy. Gyürky et al. Phys. Rev. C 68 (2003) 055803.

[15] Gy. Gyürky et al. Phys. Rev. C 64 (2001) 065803.

[16] T. Sauter, F. Kappeler, Phys. Rev. C 55 (1997) 3127.

[17] F. R. Chloupek et al. Nucl. Phys. A 652 (1999) 391.

[18] J. Bork et al. Phys. Rev. C 58 (1998) 524.

[19] Gy. Gyürky et al. Eur. Phys. J. A Direct 27 (2006) 141.

[20] W. Rapp et al. Phys. Rev. C 66 (2002) 015803.

[21] Gy. Gyürky et al. Phys. Rev. C 74 (2006) 025805.

[22] N. Özkan et al. Eur. Phys. J. A Direct 27 (2006) 145.

[23] E. Somorjai et al. Astron. Astrophys. 333 (1998) 1112.

[24] T. Rauscher, F. K. Thielemann, At. Data Tables 79 (2001) 47.

[25] A. Palumbo et al. Annual Meeting of the Division of Nuclear Physics, Nashville, Tennessee, 2006. 